\title{
Blood type and outcomes in patients with COVID-19
}

\author{
Christopher A. Latz ${ }^{1}$ (D) Charles DeCarlo $^{1} \cdot$ Laura Boitano $^{1} \cdot$ C. Y. Maximilian Png ${ }^{1} \cdot$ Rushad Patell $^{2} \cdot$ Mark F. Conrad $^{1}$. \\ Matthew Eagleton ${ }^{1} \cdot$ Anahita Dua ${ }^{1}$
}

Received: 21 June 2020 / Accepted: 6 July 2020 / Published online: 12 July 2020

(C) Springer-Verlag GmbH Germany, part of Springer Nature 2020

\begin{abstract}
This study aimed to determine if there is an association between ABO blood type and severity of COVID-19 defined by intubation or death as well as ascertain if there is variability in testing positive for COVID-19 between blood types. In a multi-institutional study, all adult patients who tested positive for COVID-19 across five hospitals were identified and included from March 6th to April 16th, 2020. Hospitalization, intubation, and death were evaluated for association with blood type. Univariate analysis was conducted using standard techniques and logistic regression was used to determine the independent effect of blood type on intubation and/or death and positive testing. During the study period, there were 7648 patients who received COVID-19 testing throughout the institutions. Of these, 1289 tested positive with a known blood type. A total of 484 (37.5\%) were admitted to hospital, $123(9.5 \%)$ were admitted to the ICU, 108 (8.4\%) were intubated, $3(0.2 \%)$ required ECMO, and $89(6.9 \%)$ died. Of the 1289 patients who tested positive, $440(34.2 \%)$ were blood type A, 201 (15.6\%) were blood type B, 61 (4.7\%) were blood type AB, and 587 (45.5\%) were blood type O. On univariate analysis, there was no association between blood type and any of the peak inflammatory markers (peak WBC, $p=0.25$; peak LDH, $p=0.40$; peak ESR, $p=0.16$; peak CRP, $p=0.14$ ) nor between blood type and any of the clinical outcomes of severity (admission $p=$ 0.20 , ICU admission $p=0.94$, intubation $p=0.93$, proning while intubated $p=0.58$, ECMO $p=0.09$, and death $p=0.49$ ). After multivariable analysis, blood type was not independently associated with risk of intubation or death (referent blood type A; blood type B: AOR: $0.72,95 \%$ CI: 0.42-1.26, blood type AB: AOR: 0.78, CI: 0.33-1.87, blood type O: AOR: 0.77, CI: 0.51-1.16), rhesus factor positive (Rh+): AOR: 1.03, CI: 0.93-1.86. Blood type A had no correlation with positive testing (AOR: 1.00, CI: 0.88-1.13), blood type $\mathrm{B}$ was associated with higher odds of testing positive for disease (AOR: 1.28, CI: 1.08-1.52), AB was also associated with higher odds of testing positive (AOR: 1.37, CI: 1.02-1.83), and O was associated with a lower risk of testing positive (AOR: 0.84, CI: $0.75-$ 0.95). $\mathrm{Rh}+$ status was associated with higher odds of testing positive (AOR: 1.23, CI: 1.003-1.50). Blood type was not associated with risk of intubation or death in patients with COVID-19. Patients with blood types $\mathrm{B}$ and $\mathrm{AB}$ who received a test were more likely to test positive and blood type $\mathrm{O}$ was less likely to test positive. $\mathrm{Rh}+$ patients were more likely to test positive.
\end{abstract}

Keywords COVID-19 $\cdot$ Blood type $\cdot$ SARS-CoV2 $\cdot$ Coronavirus

\section{Introduction}

COVID-19, the disease caused by the SARS-COV2 virus, has led to a global pandemic $[1,2]$. The SARS-COV2 virus has

Christopher A. Latz and Charles DeCarlo are Co-first authors

Christopher A. Latz

christopher.latz@mgh.harvard.edu

1 Division of Vascular and Endovascular Surgery, Department of Surgery, Massachusetts General Hospital (MGH), 15 Parkman Street, Boston, MA 02214-3117, USA

2 Division of Hematology, Department of Medicine, Beth Israel Deaconess Medical Center (BIDMC), Boston, MA, USA had varying effects on the global population; those who are older and with comorbidities such as cardiovascular disease, diabetes, and pulmonary diseases have proven more vulnerable to severe disease [3-7]. Given the significant morbidity and mortality associated with COVID-19, there has been scientific interest in eliciting data that details characteristics that may render individuals more susceptible to COVID-19 infection and determining what risk factors may be associated with progression and severity of disease from the virus [8-11].

There have been numerous molecular level hypotheses raised for the variable susceptibility to disease and vulnerability to severe disease, such as the variable expression of ACE-2 expression in the airway epithelia [12]. Landsteiner's ABO 
carbohydrate moieties are genetically inherited and previous reports have suggested a correlation between $\mathrm{ABO}$ blood type, cardiovascular disease, and cancers, as well as typing and susceptibility to certain infections, including SARS coronavirus [13-18]. In currently pre-printed data, Zhao et al. reported a possible association between blood type A and a higher risk for COVID-19 infection and mortality while blood group $\mathrm{O}$ was associated with a lower risk of infection and mortality [13]. Zietz and Tatonetti found that blood type A was correlated with a higher odds of testing positive for disease [19].

There is a paucity of data regarding the relationship between ABO blood typing and severity of COVID-19 disease. Using a large multi-institutional cohort of patients, this study aimed to determine if there is an association between $\mathrm{ABO}$ blood type and severity of COVID-19 disease as well as ascertain if there is variability in testing positive for COVID-19 between blood types.

\section{Methods}

Multi-institutional data were retrospectively reviewed on all patients with COVID-19 who presented to five major hospitals in the state of Massachusetts from March 6th to April 16th, 2020. The Partner's Healthcare System's Research Patient Data Registry (RPDR) was queried to document all patients who had a COVID-19 test performed in within the study period. Patients who tested positive for COVID-19 and had a blood type recorded in the health records were included in this study. Demographics, comorbidities, and laboratory markers of inflammation were reviewed. Patients less than 18 years of age were excluded. This study was reviewed and approved by the Massachusetts General Hospital institutional review board (IRB), and the requirement for individual informed consent was waived. All procedures followed were in accordance with the ethical standards of the responsible committee on human experimentation (both institutional and national) and with the Helsinki Declaration of 1975, as revised in 2008.

\section{Definitions}

Patient baseline comorbidities were determined by the International Classification of Diseases Codes, Ninth and Tenth Revision (ICD-9/10) which were assigned prior to the study period; all patients within the cohort had codes available prior to the study period. Comorbidities of interest were analyzed as binary variables and included hypertension, smoking (former or current), hyperlipidemia, chronic obstructive pulmonary disease (COPD), cardiovascular comorbidities (history of coronary artery disease and stroke history), diabetes mellitus, a history of cancer, chronic kidney disease (CKD), end stage renal disease on hemodialysis (ESRD), cirrhosis, cardiac dysrhythmia, deep vein thrombosis (DVT), pulmonary embolism, and asthma. Patients were recorded as taking medications of interest if that medication was recorded on the medication list at an encounter within the year prior to a positive COVID-19 test. Medications of interest included aspirin, p2y12 inhibitor, anticoagulants, antihypertensives, and beta-blockers. Initial and peak lab values were recorded for creatinine, $\mathrm{C}$-reactive protein (CRP), erythrocyte sedimentation rate (ESR), lactate dehydrogenase (LDH), and white blood cell count (WBC). Age and BMI were treated as continuous variables.

The primary outcome of interest was the composite of intubation or death. These occurrences, along with hospital admission, intensive care unit (ICU) admission, prone positioning while intubated, and initiation of extracorporeal membrane oxygenation (ECMO), were determined by manual chart review. Patient peak inflammatory marker values (WBC, LDH, CRP, and ESR) were also evaluated for correlation with blood type. Patients were followed until April 29th, 2020.

Proning referred only to proning while ventilated and not proning when not ventilated. Admission referred to admission in which the primary purpose for admission was for COVID19 infection or any admission where treatment specifically related to COVID-19 took place. Death was defined as any mortality in which COVID-19 infection complications played a role in patient death.

\section{Statistical analysis}

Continuous variables were evaluated for normality as determined by skewness and kurtosis. The Chi-square test was utilized for categorical variables, and the ANOVA or Kruskal-Wallis was used, where appropriate, in comparing demographics, comorbidities, and medications across blood types. A univariate screen between blood type, demographics, comorbidities, and the outcomes of interest were performed using the Chi-square test for categorical variables and the Student's $t$ test or the Wilcoxon ranksum for continuous variables, as appropriate. Multivariable analysis was performed for the intubation and death composite variable using logistic regression; the model was built using a purposeful selection method with blood type and rhesus factor $(\mathrm{Rh}+)$ to be forced into the model at its completion, should they not already be selected for model inclusion. Purposeful selection inclusion criteria were $P$ value $<.20$ on univariate analysis as the threshold for model inclusion; exit criteria was $P>.10$. Primary language, sex, age, and $\mathrm{Rh}+$ were determined for adjustment as these covariates were thought to be potential confounders 
Table 1 Demographics and patient comorbidities. $N$ total $=1289$. BMI: body mass index, COPD: chronic obstructive pulmonary disease, MI: myocardial infarction, ESRD: end-stage renal disease, CAD: coronary artery disease, CKD: chronic kidney disease, DVT: deep venous thrombosis, PE: pulmonary embolism, DOAC: direct oral anticoagulant. White refers to non-white, non-Hispanic

\begin{tabular}{|c|c|c|c|c|c|c|}
\hline Factor & Level & Blood type A & Blood type B & Blood type $\mathrm{AB}$ & Blood type O & $P$ value \\
\hline$N$ & & 440 & 201 & 61 & 587 & \\
\hline Age, mean (SD) & & $56.9(18.6)$ & $57.6(18.1)$ & $57.1(19.9)$ & $54.8(18.1)$ & 0.14 \\
\hline BMI, mean (SD) & & $30.8(6.5)$ & $30.6(6.7)$ & $29.4(5.4)$ & $32.0(14.9)$ & 0.32 \\
\hline Rhesus positive & & $392(89.1 \%)$ & $183(91.0 \%)$ & $53(86.9 \%)$ & $533(90.8 \%)$ & 0.63 \\
\hline Female sex & & $299(68.0 \%)$ & $136(67.7 \%)$ & $33(54.1 \%)$ & $404(68.8 \%)$ & 0.14 \\
\hline \multirow[t]{3}{*}{ Language (primary) } & English & $328(74.5 \%)$ & $149(74.1 \%)$ & $54(88.5 \%)$ & $382(65.1 \%)$ & $<0.001$ \\
\hline & Spanish & $88(20.0 \%)$ & $36(17.9 \%)$ & $4(6.6 \%)$ & $180(30.7 \%)$ & \\
\hline & Other & $24(5.5 \%)$ & $16(8.0 \%)$ & $3(4.9 \%)$ & $25(4.3 \%)$ & \\
\hline \multirow[t]{5}{*}{ Race } & White & $221(50.2 \%)$ & $80(39.8 \%)$ & $28(45.9 \%)$ & $224(38.2 \%)$ & 0.008 \\
\hline & Black & $84(19.1 \%)$ & $49(24.4 \%)$ & $15(24.6 \%)$ & $114(19.4 \%)$ & \\
\hline & Hispanic & $52(11.8 \%)$ & $25(12.4 \%)$ & $4(6.6 \%)$ & $103(17.5 \%)$ & \\
\hline & Other & $77(17.5 \%)$ & $41(20.4 \%)$ & $13(21.3 \%)$ & $128(21.8 \%)$ & \\
\hline & Not Reported & $6(1.4 \%)$ & $6(3.0 \%)$ & $1(1.6 \%)$ & $18(3.1 \%)$ & \\
\hline Hypertension & & $256(58.2 \%)$ & $124(61.7 \%)$ & $42(68.9 \%)$ & $341(58.1 \%)$ & 0.34 \\
\hline Smoker & & $97(22.0 \%)$ & $39(19.4 \%)$ & $15(24.6 \%)$ & $117(19.9 \%)$ & 0.69 \\
\hline Hyperlipidemia & & $251(57.0 \%)$ & $105(52.2 \%)$ & $35(57.4 \%)$ & $323(55.0 \%)$ & 0.70 \\
\hline COPD & & $55(12.5 \%)$ & $26(12.9 \%)$ & $9(14.8 \%)$ & $72(12.3 \%)$ & 0.95 \\
\hline Diabetes mellitus & & $150(34.1 \%)$ & $66(32.8 \%)$ & $25(41.0 \%)$ & $197(33.6 \%)$ & 0.68 \\
\hline Cancer diagnosis & & $131(29.8 \%)$ & $57(28.4 \%)$ & $19(31.1 \%)$ & $161(27.4 \%)$ & 0.83 \\
\hline Cirrhosis & & $14(3.2 \%)$ & $9(4.5 \%)$ & $1(1.6 \%)$ & $21(3.6 \%)$ & 0.72 \\
\hline Asthma & & $124(28.2 \%)$ & $46(22.9 \%)$ & $14(23.0 \%)$ & $163(27.8 \%)$ & 0.44 \\
\hline History of stroke & & $50(11.4 \%)$ & $30(14.9 \%)$ & $10(16.4 \%)$ & $71(12.1 \%)$ & 0.47 \\
\hline ESRD & & $17(3.9 \%)$ & $13(6.5 \%)$ & $5(8.2 \%)$ & $30(5.1 \%)$ & 0.33 \\
\hline CAD & & $171(38.9 \%)$ & $71(35.3 \%)$ & $30(49.2 \%)$ & $231(39.4 \%)$ & 0.28 \\
\hline CKD & & $74(16.8 \%)$ & $41(20.4 \%)$ & $12(19.7 \%)$ & $111(18.9 \%)$ & 0.70 \\
\hline Dysrhythmia & & $199(45.2 \%)$ & $95(47.3 \%)$ & $34(55.7 \%)$ & $274(46.7 \%)$ & 0.49 \\
\hline Congestive heart failure & & $71(16.1 \%)$ & $41(20.4 \%)$ & $13(21.3 \%)$ & $107(18.2 \%)$ & 0.51 \\
\hline History DVT & & $39(8.9 \%)$ & $14(7.0 \%)$ & $6(9.8 \%)$ & $43(7.3 \%)$ & 0.71 \\
\hline History PE & & $26(5.9 \%)$ & $10(5.0 \%)$ & $4(6.6 \%)$ & $24(4.1 \%)$ & 0.55 \\
\hline Aspirin & & $66(15.0 \%)$ & $45(22.4 \%)$ & $16(26.2 \%)$ & $96(16.4 \%)$ & 0.029 \\
\hline Warfarin & & $14(3.2 \%)$ & $7(3.5 \%)$ & $1(1.6 \%)$ & $20(3.4 \%)$ & 0.90 \\
\hline Statin use & & $132(30.0 \%)$ & $64(31.8 \%)$ & $25(41.0 \%)$ & $141(24.0 \%)$ & 0.007 \\
\hline Calcium channel blocker & & $56(12.7 \%)$ & $29(14.4 \%)$ & $5(8.2 \%)$ & $76(12.9 \%)$ & 0.65 \\
\hline Thiazide diuretic & & $36(8.2 \%)$ & $14(7.0 \%)$ & $4(6.6 \%)$ & $46(7.8 \%)$ & 0.94 \\
\hline ACE inhibitor & & $59(13.4 \%)$ & $32(15.9 \%)$ & $7(11.5 \%)$ & $81(13.8 \%)$ & 0.78 \\
\hline ARB & & $32(7.3 \%)$ & $19(9.5 \%)$ & $6(9.8 \%)$ & $50(8.5 \%)$ & 0.76 \\
\hline Beta blocker & & $97(22.0 \%)$ & $51(25.4 \%)$ & $14(23.0 \%)$ & $124(21.1 \%)$ & 0.66 \\
\hline DOAC & & $31(7.0 \%)$ & $12(6.0 \%)$ & $6(9.8 \%)$ & $23(3.9 \%)$ & 0.071 \\
\hline p2y12 inhibitor & & $11(2.5 \%)$ & $11(5.5 \%)$ & $4(6.6 \%)$ & $9(1.5 \%)$ & 0.006 \\
\hline
\end{tabular}

based on the primary model and these data were available for all patients who tested positive or negative. The model was run for each blood type against all others and with blood type as categorical to evaluate the independent effect of Rh+ status. Model diagnostics were performed using the Hosmer and Lemeshow Goodness of Fit test. An alpha level of $\leq .05$ was utilized as the threshold for statistical significance. There were no missing data for any demographics, comorbidities, or medications. Statistical analysis for inflammatory marker outcomes included only patients who had such tests. All analyses were performed using Stata version 15.1 (StataCorp, College station, Texas, USA). 
Table 2 Univariate analysis

\begin{tabular}{llllll}
\hline Peak creatinine & $1.8(2.3)$ & $1.9(2.4)$ & $1.5(1.7)$ & $1.7(2.2)$ & 0.64 \\
Peak WBC, mean (SD) & $9.9(5.9)$ & $10.2(12.1)$ & $10.9(7.9)$ & $8.9(5.9)$ & 0.25 \\
Peak LDH, mean (SD) & $484.8(1180.4)$ & $414.1(198.0)$ & $324.3(141.3)$ & $375.5(165.0)$ & 0.40 \\
Peak ESR, mean (SD) & $64.5(37.9)$ & $63.3(36.4)$ & $63.7(37.9)$ & $55.7(33.4)$ & 0.16 \\
Peak CRP, mean (SD) & $139.3(110.3)$ & $140.7(97.8)$ & $139.0(116.5)$ & $118.0(95.3)$ & 0.14 \\
Admitted & $158(35.9 \%)$ & $85(42.3 \%)$ & $28(45.9 \%)$ & $213(36.3 \%)$ & 0.20 \\
ICU admission & $41(9.3 \%)$ & $18(9.0 \%)$ & $7(11.5 \%)$ & $57(9.7 \%)$ & 0.94 \\
Intubated & $38(8.6 \%)$ & $15(7.5 \%)$ & $6(9.8 \%)$ & $49(8.3 \%)$ & 0.93 \\
Required proning & $18(4.1 \%)$ & $4(2.0 \%)$ & $2(3.3 \%)$ & $23(3.9 \%)$ & 0.58 \\
ECMO & $1(0.2 \%)$ & $2(1.0 \%)$ & $0(0.0 \%)$ & $0(0.0 \%)$ & 0.088 \\
Dead & $36(8.2 \%)$ & $14(7.0 \%)$ & $5(8.2 \%)$ & $34(5.8 \%)$ & 0.49 \\
Intubation/death (ID) & $63(14.3)$ & $23(11.4)$ & $8(13.1)$ & $68(11.6)$ & 0.57 \\
\hline
\end{tabular}

$W B C$ white blood count, $L D H$ lactate dehydrogenase, ESR erythrocyte sediment rate, CRP C-reactive protein, ICU Intensive Care Unit, ECMO extracorporeal membrane oxygenation

\section{Results}

During the study period, there were 7648 symptomatic patients who received a COVID-19 test throughout the five institutions included. Of these, 1289 tests were positive and had their blood group documented hence included in the analysis; the demographics, comorbidities, and medications of this population are outlined in Table 1. Of these, 484 (37.5\%) were admitted to hospital, $123(9.5 \%)$ were admitted to the ICU, $108(8.4 \%)$ were intubated, $3(0.2 \%)$ required ECMO, and 89 (6.9\%) died. Of the 1289 patients who tested positive, 440 (34.2\%) were blood type A, 201 (15.6\%) were blood type B, 61 (4.7\%) were blood type AB, and 587 (45.5\%) were blood type O. Six hundred and four COVID-19 positive patients had their WBC recorded (blood type A: 204, blood type B: 104, blood type AB: 35, blood type O: 261); 511 had their LDH evaluated (blood type A: 169, blood type B: 91, blood type AB: 29, blood type O: 222); 487 had their CRP evaluated (blood type A: 85 , blood type B: 28, blood type AB: 212, blood type O: 487); and 393 had their ESR evaluated (blood

Table 3 Multivariable analysis: blood type versus intubation/death. Referent is blood type A. Also adjusted for sex, primary language, aspirin use, calcium channel blocker use, diagnoses of chronic kidney disease, coronary artery disease, prior stroke and diabetes mellitus, race not reported (referent: white), sex and presence of rhesus factor. $\mathrm{Rh}+$ : Rhesus factor positive. Hosmer and Lemeshow goodness of fit $p=0.98$

\begin{tabular}{llll}
\hline Blood type & AOR & $95 \%$ CI & $P$ value \\
\hline A & Ref & & \\
B & 0.72 & $0.42-1.26$ & 0.25 \\
$\mathrm{AB}$ & 0.78 & $0.33-1.87$ & 0.58 \\
$\mathrm{O}$ & 0.77 & $0.51-1.16$ & 0.21 \\
$\mathrm{Rh}+$ & 1.03 & $0.93-1.86$ & 0.10 \\
\hline
\end{tabular}

type A: 130, blood type B: 71, blood type AB: 28, blood type O: 164). There was no association between blood type and any of the peak inflammatory markers (peak WBC, $p=0.25$; peak LDH, $p=0.40$; peak ESR, $p=0.16$; peak CRP, $p=$ $0.14)$. Moreover, there was no association between any of the clinical outcomes (admission $p=0.20$, ICU admission $p=0.94$, intubation $p=0.93$, required proning while intubated $p=0.58$, requiring ECMO $p=0.09$, and death $p=$ 0.49 , Table 2).

In the multivariable analysis, blood type was not determined to be independently associated with COVID-19 disease severity (blood type A: ref., blood type B: AOR: 0.72, 95\% CI: $0.42-1.26$, blood type AB: AOR: $0.78,95 \%$ CI: $0.33-$ 1.87, blood type O: AOR: $0.77,95 \%$ CI: $0.51-1.16), \mathrm{Rh}+$ : AOR: 1.03 , 95\% CI: 0.93-1.86) (Table 3).

For the analysis evaluating for correlation of blood type with a positive test, blood type A had 440 (16.6\%) positive tests, blood type B had 201 (19.4\%) positive tests, blood type $\mathrm{AB}$ had 61 (19.8\%) positive tests, and blood type $\mathrm{O}$ had 587 $(16.1 \%)$ positive tests $(p=0.036)$. After multivariable

Table 4 Rate of positive test by blood type. Overall $P$ value 0.036 derived by Chi-squared testing. Adjusted odds ratio (AOR) with adjustment for sex, primary language, age, and rhesus factor with each blood type compared to all others. $\mathrm{Rh}+$ model included blood type as a categorical covariate. Rh+: Rhesus factor positive. Hosmer and Lemeshow goodness of fit $p>.5$ for all models

\begin{tabular}{lllll}
\hline Blood type & Total $N$ & $N$ positive (\%) & AOR (95\% CI) & $P$ value \\
\hline A & 2649 & $440(16.6)$ & $1.00(0.88-1.13)$ & 0.96 \\
B & 1035 & $201(19.4)$ & $1.28(1.08-1.52)$ & 0.004 \\
AB & 308 & $61(19.8)$ & $1.37(1.02-1.83)$ & 0.035 \\
O & 3656 & $587(16.1)$ & $0.84(0.75-0.95)$ & 0.007 \\
Rh+ & 6707 & $1161(17.3)$ & $1.22(1.003-1.50)$ & 0.047 \\
All & 7648 & $1289(16.9)$ & & \\
\hline
\end{tabular}


analysis, blood type A had no correlation with positive testing (AOR: 1.00, 95\% CI: 0.88-1.13), blood type B was associated with higher odds of testing positive for disease (AOR: 1.28, 95\% CI: 1.08-1.52), AB was also associated with higher odds of testing positive (AOR: 1.37, 95\% CI: 1.02-1.83), O was associated with lower odds of testing positive (AOR: 0.84, 95\% CI: 0.75-0.95), and $\mathrm{Rh}+$ blood was associated with a higher odds of testing positive (AOR: 1.22 (1.003-1.50) (Table 4).

\section{Discussion}

In this large, multi-institutional, retrospective review, there was no association noted between ABO blood type and COVID-19 disease severity defined as intubation or death. These data are different from that of Zhao et al. in the Wuhan experience who evaluated the association between blood type and mortality [13]. While there is data that $\mathrm{ABO}$ blood typing plays a role in disease acquisition and severity in other diseases, this was not the case in these data for COVID-19 [17-19]. The primary outcome of interest, composite intubation, or death yielded no association with blood type; furthermore, there were no significant associations with blood type and need for hospitalization, proning requirement while intubated or for any of the inflammatory markers reviewed in this study on univariate analysis. Given the lack of association between $\mathrm{ABO}$ subtype and severe disease found in these data as well as the preliminary data from Zietz and Tatonetti, ABO blood typing should not currently be considered prognostic in those who acquire the disease.

Blood type $\mathrm{O}$ had the lowest frequency of disease positivity, similar to Zhao et al., but blood type A had a lower frequency than blood types B and AB [13]. Both Zhao et al. and Zietz and Tatonetti, in their preliminary data, show correlation between blood type A and likelihood of positive testing and blood type $\mathrm{O}$ and likelihood of negative testing $[13,14]$. The finding related to blood type $\mathrm{O}$ appears to correlate across our study and that of both Zhao et al. and Zietz and Tatonetti, but the blood type A correlation was not found in our study [13, 14]. This association of blood type $\mathrm{O}$ being less common in infection is the same as that found for SARS-CoV-1 [17]. The $\mathrm{Rh}+$ association with disease positivity appears to be a novel finding and warrants further investigation. Given the relative rarity of rhesus negative blood types, these could not be stratified out by blood type given our patient numbers in this study.

A final element worthy of discussion is that there is certainly a racial element to $\mathrm{ABO}$ blood typing [20]. We were able to account for confounding factors of race and primary language through our multivariable models, likely isolating the effect of ABO blood typing, independent of ethnicity. However, the full effects of ethnicity on COVID-19 susceptibility and severity warrant further investigation.

\section{Limitations}

Our sample sizes are relatively small, with 483 hospitalized patients available for review and 1289 total positive patients that had blood type documented. It is possible that there may be a lead-time bias as some of the positive patients were early in their course and will go on to require hospitalization, require intubation, or die secondary to their disease. Additionally, this is an observational study, and while attempts were made to control for confounding, where such controls were possible, there is always the possibility that unmeasured confounding is driving the results.

\section{Conclusions}

Blood type is not associated with risk of progression to severe disease requiring intubation or causing death, nor is it associated with higher peak levels of inflammatory markers. Patients with blood types $\mathrm{B}$ and $\mathrm{AB}$ who received a test were more likely to test positive as were those who are $\mathrm{Rh}+$ positive, and blood type $\mathrm{O}$ was less likely to test positive.

Authors' contributions C.L. and A.D. created the concept and wrote the paper. C.L. and C.D. performed the statistical analysis and analyzed the data. C.L., C.D., L.B., C.P. performed raw data collection. C.L., C.D., L.B., C.P., R.P., M.C., M.E., and A.D assisted with interpretation of the data and critical revision of the manuscript.

\section{Compliance with ethical standards}

Conflict of interest The authors declare that they have no conflict of interest.

\section{References}

1. Rothan HA, Byrareddy SN (2020) The epidemiology and pathogenesis of coronavirus disease (COVID-19) outbreak. J Autoimmun 109:102433

2. Ahn DG, Shin HJ, Kim MH, Lee S, Kim HS, Myoung J, Kim BT, Kim SJ (2020) Current status of epidemiology, diagnosis, therapeutics, and vaccines for novel coronavirus disease 2019 (COVID-19). J Microbiol Biotechnol 30(3):313-324

3. Shi Y, Yu X, Zhao H, Wang H, Zhao R, Sheng J (2020) Host susceptibility to severe COVID-19 and establishment of a host risk score: findings of 487 cases outside Wuhan. Crit Care 24(1):108

4. Li B, Yang J, Zhao F, Zhi L, Wang X, Liu L, Bi Z, Zhao Y (2020) Prevalence and impact of cardiovascular metabolic diseases on COVID-19 in China. Clin Res Cardiol 109(5):531-538

5. von der Thüsen J, van der Eerden M (2020) Histopathology and genetic susceptibility in COVID-19 pneumonia. Eur J Clin Invest. https://oi.org/10.1111/eci.13259

6. Tian S, Hu N, Lou J, Chen K, Kang X, Xiang Z et al (2020) Characteristics of COVID-19 infection in Beijing. J Inf Secur 80(4):401-406

7. Loscertales MP, Owens S, O'Donnell J, Bunn J, Bosch-Capblanch $\mathrm{X}$, Brabin BJ (2007) ABO blood group phenotypes and 
plasmodium falciparum malaria: unlocking a pivotal mechanism. Adv Parasitol 65:1-50

8. Meng J, Xiao G, Zhang J, He X, Ou M, Bi J, Yang R, di W, Wang Z, Li Z, Gao H, Liu L, Zhang G (2020) Renin-angiotensin system inhibitors improve the clinical outcomes of COVID-19 patients with hypertension. Emerg Microbes Infect 9(1):757-760

9. Hussain A, Bhowmik B, do Vale Moreira NC (2020) COVID-19 and diabetes: knowledge in progress. Diabetes Res Clin Pract 162: 108142

10. Guo W, Li M, Dong Y, et al (2020) Diabetes is a risk factor for the progression and prognosis of COVID-19 [published online ahead of print, 2020 Mar 31]. Diabetes Metab Res Rev. https://doi.org/10. 1002/dmrr.3319

11. Zhao Q, Meng M, Kumar R, et al (2020) The impact of COPD and smoking history on the severity of COVID-19: A systemic review and meta-analysis. J Med Virol. https://doi.org/10.1002/jmv.25889

12. Leung JM, Yang CX, Tam A, Shaipanich T, Hackett TL, Singhera GK, Dorscheid DR, Sin DD (2020) ACE-2 expression in the small airway epithelia of smokers and COPD patients: implications for COVID-19. Eur Respir J 55:2000688

13. Zhao J, Yang Y, Huang H, Li D, Gu D, Lu X, Zhang Z, Liu L, Liu T, Liu Y, He Y, Sun B, Wei M, Yang G, Wang X, Zhang L, Zhou X, Xing M, Wang PG (2020) Relationship between the ABO blood group and the COVID-19 susceptibility. medRxiv. https://doi.org/ 10.1101/2020.03.11.20031096
14. Zietz M, Tatonetti N (2020) Testing the association between blood type and COVID-19 infection, intubation and death. medRxiv Preprint. https://doi.org/10.1101/2020040820058073

15. Batool Z, Durrani SH, Tariq S (2017) Association of abo and Rh blood group types to hepatitis $\mathrm{B}$, hepatitis $\mathrm{C}$, Hiv and syphilis infection, a five year' experience in healthy blood donors in a tertiary care hospital. J Ayub Med Coll Abbottabad 29(1):90-92

16. Lindesmith L, Moe C, Marionneau S, Ruvoen N, Jiang X, Lindblad L, Stewart P, LePendu J, Baric R (2003) Human susceptibility and resistance to Norwalk virus infection. Nat Med 9(5):548-553

17. Cheng Y, Cheng G, Chui CH, Lau FY, Chan PK, Ng MH, Sung JJ, Wong RS (2005) ABO blood group and susceptibility to severe acute respiratory syndrome. Jama. 293(12):1450-1451

18. Liumbruno GM, Franchini M (2013) Beyond immunohaematology: the role of the $\mathrm{ABO}$ blood group in human diseases. Blood Transfus 11(4):491-499

19. Harris JB, LaRocque RC (2016) Cholera and ABO blood group: understanding an ancient association. Am J Trop Med Hyg 95(2): 263-264

20. Liu J, Zhang S, Wang Q, Shen H, Zhang Y, Liu M (2017) Frequencies and ethnic distribution of $\mathrm{ABO}$ and $\mathrm{RhD}$ blood groups in China: a population-based cross-sectional study. BMJ Open 7(12): 018476

Publisher's note Springer Nature remains neutral with regard to jurisdictional claims in published maps and institutional affiliations. 